S. Murai and P. Singla

Nagoya Math. J.

Vol. 190 (2008), 35-61

\title{
RIGIDITY OF LINEAR STRANDS AND GENERIC INITIAL IDEALS
}

\author{
SATOSHI MURAI AND POOJA SINGLA
}

\begin{abstract}
Let $K$ be a field, $S$ a polynomial ring and $E$ an exterior algebra over $K$, both in a finite set of variables. We study rigidity properties of the graded Betti numbers of graded ideals in $S$ and $E$ when passing to their generic initial ideals. First, we prove that if the graded Betti numbers $\beta_{i i+k}^{S}(S / I)=\beta_{i i+k}^{S}(S / \operatorname{Gin}(I))$ for some $i>1$ and $k \geq 0$, then $\beta_{q q+k}^{S}(S / I)=\beta_{q q+k}^{S}(S / \operatorname{Gin}(I))$ for all $q \geq i$, where $I \subset S$ is a graded ideal. Second, we show that if $\beta_{i i+k}^{E}(E / I)=\beta_{i i+k}^{E}(E / \operatorname{Gin}(I))$ for some $i>1$ and $k \geq 0$, then $\beta_{q q+k}^{E}(E / I)=\beta_{q q+k}^{E}(E / \operatorname{Gin}(I))$ for all $q \geq 1$, where $I \subset E$ is a graded ideal. In addition, it will be shown that the graded Betti numbers $\beta_{i i+k}^{R}(R / I)=\beta_{i i+k}^{R}(R / \operatorname{Gin}(I))$ for all $i \geq 1$ if and only if $I_{\langle k\rangle}$ and $I_{\langle k+1\rangle}$ have a linear resolution. Here $I_{\langle d\rangle}$ is the ideal generated by all homogeneous elements in $I$ of degree $d$, and $R$ can be either the polynomial ring or the exterior algebra.
\end{abstract}

\section{Introduction}

In this paper we study rigidity properties of graded Betti numbers of a graded ideal when passing to its generic initial ideal.

Let $S=K\left[x_{1}, \ldots, x_{n}\right]$ be the polynomial ring in $n$ variables over a field $K$ with $\operatorname{char}(K)=0$ and $I \subset S$ a graded ideal. Let $\beta_{i}^{S}(M)=$ $\operatorname{dim}_{K} \operatorname{Tor}_{i}^{S}(K, M)$ and $\beta_{i j}^{S}(M)=\operatorname{dim}_{K} \operatorname{Tor}_{i}^{S}(K, M)_{j}$ denote respectively the $i$-th total and $i, j$-th graded Betti number of a finitely generated graded $S$ module $M$.

The generic initial ideal Gin $(I)$ plays a fundamental role in investigating various homological, algebraic, combinatorial and geometric properties of $I$. By definition, the generic initial ideal $\operatorname{Gin}(I)$ is, after performing a generic change of coordinates, the initial ideal of $I$ with respect to the reverse lexicographic order. Here we consider the reverse lexicographic order induced by $x_{1}>\cdots>x_{n}$.

Received August 28, 2006.

2000 Mathematics Subject Classification: 13D02.

The first author is supported by JSPS Research Fellowships for Young Scientists. 
The following inequality of the graded Betti numbers is well-known:

$$
\beta_{i j}(S / I) \leq \beta_{i j}(S / \operatorname{Gin}(I)),
$$

for all $i, j$ (see [9, Theorem 1.1]). Equality holds for all $i$ and $j$ if and only if $I$ is componentwise linear (see [4, Theorem 1.1]). In his paper [9] Conca asked whether the equality $\beta_{i}(S / I)=\beta_{i}(S / \operatorname{Gin}(I))$ for some $i \geq 1$ of the total Betti numbers implies $\beta_{j}(S / I)=\beta_{j}(S / \operatorname{Gin}(I))$ for all $j \geq i$. This question of Conca was positively answered in 2004 by Conca, Herzog and Hibi in [10].

One of the main results of our paper is to extend this result of ConcaHerzog-Hibi to graded Betti numbers. In Corollary 2.3 we show the following: If for some $i>1$ and $k \geq 0$, we have $\beta_{i i+k}^{S}(S / I)=\beta_{i i+k}^{S}(S / \operatorname{Gin}(I))$, then

$$
\beta_{q q+k}^{S}(S / I)=\beta_{q q+k}^{S}(S / \operatorname{Gin}(I)) \text { for all } q \geq i .
$$

We also study the same property for generic initial ideals over an exterior algebra. Let $K$ be an infinite field, $V$ an $n$-dimensional $K$-vector space with basis $e_{1}, \ldots, e_{n}$ and $E=\bigoplus_{k=0}^{n} \bigwedge^{k} V$ the exterior algebra of $V$. For a graded ideal $J \subset E$, we write $\operatorname{Gin}(J)$ for the generic initial ideal of $J$ with respect to the reverse lexicographic order induced by $e_{1}>\cdots>e_{n}$ and denote by $\beta_{i j}^{E}(E / J)$ the $i, j$-th graded Betti number of $E / J$ over $E$. Somewhat surprisingly, the following stronger property is true in the exterior algebra: If $\beta_{i i+k}^{E}(E / J)=\beta_{i i+k}^{E}(E / \operatorname{Gin}(J))$ for some $i>1$ and $k \geq 0$, then one has

$$
\beta_{q q+k}^{E}(E / J)=\beta_{q q+k}^{E}(E / \operatorname{Gin}(J)) \quad \text { for all } q \geq 1 .
$$

Let $R$ be either a polynomial ring over a field $K$ with $\operatorname{char}(K)=0$ or an exterior algebra over an infinite field and $I$ a graded ideal of $R$. The above property leads us to ask when a graded ideal $I \subset R$ satisfies $\beta_{i i+k}^{R}(R / I)=$ $\beta_{i i+k}^{R}(R / \operatorname{Gin}(I))$ for all $i \geq 1$, where we fix an integer $k \geq 0$. We will prove the following result answering this question.

THEOREM 0.1. Let $R$ be either a polynomial ring over a field $K$ with $\operatorname{char}(K)=0$ or an exterior algebra over an infinite field, $I \subset R$ a graded ideal and $k \geq 0$ an integer. The following conditions are equivalent.

(i) $\beta_{i i+k}^{R}(R / I)=\beta_{i i+k}^{R}(R / \operatorname{Gin}(I))$ for all $i \geq 1$;

(ii) $I_{\langle k\rangle}$ and $I_{\langle k+1\rangle}$ have a linear resolution;

(iii) $\beta_{1 k+1}^{R}(R / I)=\beta_{1 k+1}^{R}(R / \operatorname{Gin}(I))$ and $\beta_{1 k+2}^{R}(R / I)=\beta_{1 k+2}^{R}(R / \operatorname{Gin}(I))$, 
where $I_{\langle k\rangle}$ denotes the ideal of $R$ generated by all homogeneous elements in $I$ of degree $k$.

The above result is a generalization of [4, Theorem 1.1], where it was shown that $\beta_{i j}^{R}(R / I)=\beta_{i j}^{R}(R / \operatorname{Gin}(I))$ for all $i, j$ if and only if $I$ is componentwise linear.

In the end of the paper, we study the Cancellation Principle for generic initial ideals [13]. We find the relation between our results for Betti numbers of a graded ideal in a polynomial ring and the Cancellation Principle for generic initial ideals.

This paper is organized as follows: In Section 1, we will give an upper bound for graded Betti numbers in terms of generic annihilator numbers by using the technique developed in [10]. In Section 2, we will generalize Conca-Herzog-Hibi's theorem for graded Betti numbers over a polynomial ring. In Section 3, some basic facts about Cartan complexes and generic annihilator numbers over an exterior algebra are studied. In Section 4, we will generalize Conca-Herzog-Hibi's theorem for graded Betti numbers over an exterior algebra. In Section 5, we will study when $I_{\langle d\rangle}$ has a linear resolution from the viewpoint of generic initial ideals and give a proof of Theorem 0.1. In Section 6, we will study the Cancellation Principle. The results in the last section are closely related to the results in Section 1.

\section{§1. An upper bound for the graded Betti numbers}

In this section, we will give an upper bound for graded Betti numbers in terms of generic graded annihilator numbers, which were introduced in [10]. Note that most of the results in this section are refinements of the results in $[10, \S 1]$. Though these results seem to be somewhat technical, they are of crucial importance for the proof of one of our main theorems in the next section.

Let $S=K\left[x_{1}, \ldots, x_{n}\right]$ be the standard graded polynomial ring over an arbitrary field $K$ and $\mathfrak{m}=\left(x_{1}, \ldots, x_{n}\right)$ the graded maximal ideal. Let $M$ be a finitely generated graded $S$-module. For each nonnegative integer $i$, the modules $\operatorname{Tor}_{i}^{S}(K, M)$ are finitely generated $K$-vector spaces. The numbers $\beta_{i}^{S}(M)=\operatorname{dim}_{K} \operatorname{Tor}_{i}^{S}(K, M)$ and $\beta_{i j}^{S}(M)=\operatorname{dim}_{K} \operatorname{Tor}_{i}^{S}(K, M)_{j}$ are called Betti numbers and graded Betti numbers of $M$, respectively. As $\beta_{i j}^{S}$ are invariants under base field extensions, from now on we may assume the field $K$ to be infinite. 
Let $y_{1}, \ldots, y_{n}$ be a sequence of generic linear forms for the module $M$. For each $p=1, \ldots, n$, the modules

$$
A_{p}=\left(y_{1}, \ldots, y_{p-1}\right) M:_{M} y_{p} /\left(y_{1}, \ldots, y_{p-1}\right)
$$

are $\mathbb{Z}$-graded $S$-modules of finite length. We define $\alpha_{p}(M)=\operatorname{dim}_{K} A_{p}$, which we call the generic annihilator numbers of $M$. We denote by $\alpha_{p, j}(M)$ the vector space dimension of the $j$ th graded component $\left(A_{p}\right)_{j}$ of $A_{p}$ which we call the generic graded annihilator numbers of $M$.

Let $H_{i}(p, M)$ be the Koszul homology $H_{i}\left(y_{1}, \ldots, y_{p} ; M\right)$ of the partial sequence $y_{1}, \ldots, y_{p}$. We set $h_{i}(p, M)=\operatorname{dim}_{K} H_{i}(p, M)$ and $h_{i j}(p, M)=$ $\operatorname{dim}_{K} H_{i}(p, M)_{j}$. We omit $M$ and simply write $\beta_{i j}^{S}, \beta_{i}^{S}, \alpha_{i, j}, \alpha_{i}, H_{i}(p)_{j}$, $H_{i}(p), h_{i j}(p), h_{i}(p)$ for the above defined terms, if the module under consideration is fixed. Then we have the following long exact sequence (see [6, Corollary 1.6.13]):

$$
\begin{aligned}
& \cdots \longrightarrow H_{i}(p-1) \stackrel{\varphi_{i, p-1}}{\longrightarrow} H_{i}(p-1) \longrightarrow H_{i}(p) \longrightarrow H_{i-1}(p-1) \\
& \cdots \longrightarrow H_{0}(p-1) \stackrel{\varphi_{0, p-1}}{\longrightarrow} H_{0}(p-1) \longrightarrow H_{0}(p) \longrightarrow 0
\end{aligned}
$$

In the above sequence $\varphi_{i, p-1}$ is the multiplication map on $H_{i}(p-1)$ with multiplication by $\pm y_{p}$. One may notice that $A_{p}$ is given by the kernel of the map $\varphi_{0, p-1}$. Hence we get the following exact sequences with all the maps of degree zero:

$$
0 \longrightarrow \operatorname{Im} \varphi_{1, p-1} \longrightarrow H_{1}(p-1) \longrightarrow H_{1}(p) \longrightarrow A_{p}(-1) \longrightarrow 0
$$

for all $p$, and

$$
\begin{aligned}
0 \longrightarrow \operatorname{Im} \varphi_{i, p-1} \longrightarrow H_{i}(p-1) & \longrightarrow H_{i}(p) \longrightarrow H_{i-1}(p-1)(-1) \\
& \longrightarrow \operatorname{Im} \varphi_{i-1, p-1} \longrightarrow 0
\end{aligned}
$$

for all $p$ and $i>1$.

Let $\delta_{i, j, k}=\operatorname{dim}_{K}\left(\operatorname{Im} \varphi_{i, j}\right)_{k}$. From the above exact sequences, we obtain the following equations for each integer $k \geq 0$ :

$$
h_{1 k}(p)=h_{1 k}(p-1)+\alpha_{p, k-1}-\delta_{1, p-1, k},
$$

and for all $i>1$,

(3) $h_{i, i+k}(p)=h_{i, i+k}(p-1)+h_{i-1, i-1+k}(p-1)-\delta_{i, p-1, i+k}-\delta_{i-1, p-1, i+k}$.

By using (2) and (3), we obtain 
Proposition 1.1. For all nonnegative integers $i \geq 1$ and $k$, one has

$$
\text { (4) } \begin{aligned}
h_{i, i+k}(p)= & \sum_{j=1}^{p-i+1}\left(\begin{array}{c}
p-j \\
i-1
\end{array}\right) \alpha_{j, k} \\
& -\sum_{(a, b) \in A_{i, p}}\left[\left(\begin{array}{c}
p-b-1 \\
i-a
\end{array}\right) \delta_{a, b, a+k}+\left(\begin{array}{c}
p-b-1 \\
i-a-1
\end{array}\right) \delta_{a, b, a+k+1}\right]
\end{aligned}
$$

where the set $A_{i, p}=\left\{(a, b) \in \mathbb{N}^{2}: 1 \leq b \leq p-1\right.$ and $\max \{i-p+b, 1\} \leq$ $a \leq i\}$

Proof. We will prove the above formula by induction on $p$. For $p=1$, we have from Equation (2) and Equation (3):

$$
h_{i, i+k}(1)= \begin{cases}\alpha_{1, k} & \text { if } i=1 \\ 0 & i \geq 2\end{cases}
$$

which is what the formula given in the statement of the proposition suggests. Now we assume $p>1$ and we assume the result to be true for $p-1$.

Let first $i=1$. By induction hypothesis and from Equation (2), we get:

$$
\begin{aligned}
& h_{1,1+k}(p)=h_{1,1+k}(p-1)+\alpha_{p, k}-\delta_{1, p-1,1+k} \\
& =\sum_{j=1}^{p-1}\left(\begin{array}{c}
p-1-j \\
0
\end{array}\right) \alpha_{j, k}-\sum_{(a, b) \in A_{1, p-1}}\left(\begin{array}{c}
p-b-2 \\
1-a
\end{array}\right) \delta_{a, b, a+k}+\alpha_{p, k}-\delta_{1, p-1,1+k} \\
& =\sum_{j=1}^{p} \alpha_{j, k}-\sum_{(a, b) \in A_{1, p}}\left[\left(\begin{array}{c}
p-b-1 \\
1-a
\end{array}\right) \delta_{a, b, a+k}\right]
\end{aligned}
$$

which is what the formula suggests.

Now let $i>1$. From Equation (3), we have:

$$
h_{i, i+k}(p)=h_{i, i+k}(p-1)+h_{i-1, i-1+k}(p-1)-\delta_{i, p-1, i+k}-\delta_{i-1, p-1, i+k} .
$$

Note that one has $\left(\begin{array}{l}a \\ b\end{array}\right)+\left(\begin{array}{c}a \\ b+1\end{array}\right)=\left(\begin{array}{l}a+1 \\ b+1\end{array}\right)$ for all integers $a \geq b \geq 0$. Then, using induction hypothesis, the right hand side of the above equation is a sum of the following three terms:

$$
\sum_{j=1}^{p-i+1}\left\{\left(\begin{array}{c}
p-j-1 \\
i-1
\end{array}\right)+\left(\begin{array}{c}
p-j-1 \\
i-2
\end{array}\right)\right\} \alpha_{j, k}=\sum_{j=1}^{p-i+1}\left(\begin{array}{c}
p-j \\
i-1
\end{array}\right) \alpha_{j, k}
$$


$\begin{aligned}(6)-\left\{\sum_{(a, b) \in A_{i, p-1}}\left(\begin{array}{c}p-b-2 \\ i-a\end{array}\right) \delta_{a, b, a+k}+\delta_{i, p-1, i+k}\right. & \\ & \left.+\sum_{(a, b) \in A_{i-1, p-1}}\left(\begin{array}{c}p-b-2 \\ i-a-1\end{array}\right) \delta_{a, b, a+k}\right\}\end{aligned}$

and

(7) $-\left\{\sum_{(a, b) \in A_{i, p-1}}\left(\begin{array}{l}p-b-2 \\ i-a-1\end{array}\right) \delta_{a, b, a+k+1}+\delta_{i-1, p-1, i+k}\right.$

$$
\left.+\sum_{(a, b) \in A_{i-1, p-1}}\left(\begin{array}{l}
p-b-2 \\
i-a-2
\end{array}\right) \delta_{a, b, a+k+1}\right\}
$$

The term (6) can be written as:

$$
\begin{aligned}
& -\left\{\sum_{(a, b) \in A_{i, p-1}}\left(\begin{array}{c}
p-b-2 \\
i-a
\end{array}\right) \delta_{a, b, a+k}\right. \\
& \left.\quad+\sum_{(a, b) \in A_{i, p-1}}\left(\begin{array}{c}
p-b-2 \\
i-a-1
\end{array}\right) \delta_{a, b, a+k}+\delta_{i, p-1, i+k}\right\}
\end{aligned}
$$

which is further equal to

$$
-\left\{\sum_{(a, b) \in A_{i, p-1}}\left(\begin{array}{c}
p-b-1 \\
i-a
\end{array}\right) \delta_{a, b, a+k}+\delta_{i, p-1, i+k}\right\},
$$

which in the end equals

$$
-\sum_{(a, b) \in A_{i, p}}\left(\begin{array}{c}
p-b-1 \\
i-a
\end{array}\right) \delta_{a, b, a+k}
$$

Now we notice that the term (7) can be written as:

$$
\begin{gathered}
-\left\{\sum_{(a, b) \in A_{i, p-1}}\left(\begin{array}{l}
p-b-2 \\
i-a-1
\end{array}\right) \delta_{a, b, a+k+1}+\sum_{(a, b) \in A_{i, p-1}}\left(\begin{array}{l}
p-b-2 \\
i-a-2
\end{array}\right) \delta_{a, b, a+k+1}\right. \\
\left.+\sum_{b=p-i+1}^{p-2} \delta_{i-p+b, b, i-p+b+k+1}+\delta_{i-1, p-1, i+k}\right\} .
\end{gathered}
$$


This can be rewritten as:

$$
\begin{aligned}
-\left\{\sum_{(a, b) \in A_{i, p-1}}\left(\begin{array}{c}
p-b-1 \\
i-a-1
\end{array}\right)\right. & \delta_{a, b, a+k+1} \\
& \left.+\sum_{b=p-i+1}^{p-2} \delta_{i-p+b, b, i-p+b+k+1}+\delta_{i-1, p-1, i+k}\right\}
\end{aligned}
$$

which then is equal to

$$
-\sum_{(a, b) \in A_{i, p}}\left(\begin{array}{l}
p-b-1 \\
i-a-1
\end{array}\right) \delta_{a, b, a+k+1} .
$$

Hence $h_{i, i+k}(p)$ is the sum of (5), (8) and (9), as required.

Remark 1.2. Notice that summing the formula stated in Proposition 1.1 over $k$, gives us back the formula given in the proof of [10, Proposition 1.1].

Proposition 1.1 implies the following fact.

Corollary 1.3. We have

(a) $h_{i, i+k}(p) \leq \sum_{j=1}^{p-i+1}\left(\begin{array}{c}p-j \\ i-1\end{array}\right) \alpha_{j, k}$.

(b) For given integers $i \geq 1$ and $p \geq 1$, the following conditions are equivalent:

(i) $h_{i, i+k}(p)=\sum_{j=1}^{p-i+1}\left(\begin{array}{c}p-j \\ i-1\end{array}\right) \alpha_{j, k}$

(ii) $\left(\operatorname{Im} \varphi_{a, b}\right)_{(a+k)}=0$ for all $(a, b) \in A_{i, p} \backslash\{(i-p+b, b): b \leq p-1\}$ and $\left(\operatorname{Im} \varphi_{a, b}\right)_{(a+k+1)}=0$ for all $(a, b) \in A_{i, p} \backslash\{(i, b): b \leq p-1\}$.

(iii) $\left(\mathfrak{m} H_{a}(b)\right)_{(a+k)}=0$ for all $(a, b) \in A_{i, p} \backslash\{(i-p+b, b): b \leq p-1\}$ and $\left(\mathfrak{m} H_{a}(b)\right)_{(a+k+1)}=0$ for all $(a, b) \in A_{i, p} \backslash\{(i, b): b \leq p-1\}$.

Proof. Statement (a) is clear from Proposition 1.1. The equivalence of (i) and (ii) follows immediately from Proposition 1.1. Indeed, $h_{i, i+k}(p)=$ $\sum_{j=1}^{p-i+1}\left(\begin{array}{c}p-j \\ i-1\end{array}\right) \alpha_{j, k}$ if and only if all graded maps appearing in the formula in Proposition 1.1 vanish whenever their binomial coefficients are nonzero. And for the equivalence of (ii) and (iii), we may notice that a generic linear form annihilates $\left(H_{a}(b)\right)_{k}$ if and only if $\mathfrak{m}$ annihilates $\left(H_{a}(b)\right)_{k}$. 
The next corollary is a special case $(p=n)$ of the above corollary.

Corollary 1.4. (a) $\beta_{i i+k}^{S} \leq \sum_{j=1}^{n-i+1}\left(\begin{array}{c}n-j \\ i-1\end{array}\right) \alpha_{j, k}$ for all $i \geq 1$.

(b) For given $i \geq 1$ the following are equivalent:

(i) $\beta_{i i+k}^{S}=\sum_{j=1}^{n-i+1}\left(\begin{array}{c}n-j \\ i-1\end{array}\right) \alpha_{j, k}$.

(ii) $\left(\operatorname{Im} \varphi_{a, b}\right)_{(a+k)}=0$ for all $(a, b) \in A_{i, n} \backslash\{(i-n+b, b), b \leq n-1\}$ and $\left(\operatorname{Im} \varphi_{a, b}\right)_{(a+k+1)}=0$ for all $(a, b) \in A_{i, n} \backslash\{(i, b), b \leq n-1\}$.

(iii) $\left(\mathfrak{m} H_{a}(b)\right)_{(a+k)}=0$ for all $(a, b) \in A_{i, n} \backslash\{(i-n+b, b), b \leq n-1\}$ and $\left(\mathfrak{m} H_{a}(b)\right)_{(a+k+1)}=0$ for all $(a, b) \in A_{i, n} \backslash\{(i, b), b \leq n-1\}$.

\section{$\S 2$. Graded rigidity of resolutions and linear components}

In this section we generalize [10, Theorem 2.3] of Conca-Herzog-Hibi. They gave an upper bound of total Betti numbers in terms of generic annihilator numbers, and proved that if the Betti number $\beta_{i}^{S}(M)$ for some $i \geq 1$ reaches its upper bound, then the Betti numbers $\beta_{q}^{S}(M)$ also reach their upper bounds for all $q \geq i$. We show that if a graded Betti number $\beta_{i i+k}^{S}(M)$ for some $i>1$ reaches its upper bound given in Corollary 1.4, then so do all the graded Betti numbers $\beta_{q q+k}^{S}(M)$ for $q \geq i$. Here we need the assumption $i>1$ as we will see later in Remark 2.4.

We state the main theorem of this section:

TheOREM 2.1. Let $M$ be a finitely generated graded $S$-module. Suppose for some $i>1$, we have $\beta_{i i+k}^{S}(M)=\sum_{j=1}^{n-i+1}\left(\begin{array}{c}n-j \\ i-1\end{array}\right) \alpha_{j, k}(M)$. Then

$$
\beta_{q q+k}^{S}(M)=\sum_{j=1}^{n-q+1}\left(\begin{array}{l}
n-j \\
q-1
\end{array}\right) \alpha_{j, k}(M) \quad \text { for all } q \geq i
$$

Before proving the theorem, we recall the following vanishing property of Koszul homology. For a sequence of elements $y_{1}, \ldots, y_{r} \in S$ and a set $A \subseteq\{1, \ldots, r\}$, we set $y_{A}=\left\{y_{j}: j \in A\right\}$.

LEMMA 2.2. Let $I \supseteq\left(y_{1}, \ldots, y_{r}\right)$ and assume that $\left(I H_{i}\left(y_{A} ; M\right)\right)_{i+k}=$ 0 for all $A \subseteq\{1, \ldots, r\}$ for some $i, k$. Then $\left(I H_{i+1}\left(y_{A}, M\right)\right)_{i+k+1}=0$ for all $A \subseteq\{1, \ldots, r\}$.

The proof of Lemma 2.2 is the same as [10, Corollary 2.3]. Hence we skip the proof. 
Proof of Theorem 2.1. First we notice that it is enough to prove the claim in the case when $q=i+1$. Therefore we only need to show that $\left(\mathfrak{m} H_{a}(b)\right)_{a+k}=0$ for all $(a, b) \in A_{i+1, n} \backslash\{(i+1-n+b, b): b \leq n-1\}$ and $\left(\mathfrak{m} H_{a}(b)\right)_{a+k+1}=0$ for all $(a, b) \in A_{i+1, n} \backslash\{(i+1, b): b \leq n-1\}$, as is clear from Corollary 1.3.

By assumption, $\left(\mathfrak{m} H_{a}(b)\right)_{a+k}=0$ for all $(a, b) \in A_{i, n} \backslash\{(i-n+b, b)$ : $b \leq n-1\}$ and $\left(\mathfrak{m} H_{a}(b)\right)_{a+k+1}=0$ for all $(a, b) \in A_{i, n} \backslash\{(i, b): b \leq n-1\}$. Also a routine computation implies

$$
A_{i+1, n} \backslash\left(A_{i, n} \backslash\{(i-n+b, b): b \leq n-1\}\right)=\{(i+1, b): b \leq n-1\}
$$

and

$$
\begin{aligned}
\left(A_{i+1, n} \backslash\{(i+1, b): b \leq n-1\}\right) \backslash\left(A_{i, n} \backslash\{(i, b)\right. & : b \leq n-1\}) \\
& =\{(i, b): b \leq n-1\} .
\end{aligned}
$$

Therefore, we need to show that $\left(\mathfrak{m} H_{i+1}(b)\right)_{i+1+k}=0$ and $\left(\mathfrak{m} H_{i}(b)\right)_{i+k+1}=$ 0 for all $b \leq n-1$. However, from assumption $\left(\mathfrak{m} H_{i}(b)\right)_{i+k}=0$ and $\left(\mathfrak{m} H_{i-1}(b)\right)_{i+k}=0$ for all $b \leq n-1$, now it follows from Lemma 2.2 that for all $b \leq n-1$, we have $\left(\mathfrak{m} H_{i+1}(b)\right)_{i+1+k}=0$ and $\left(\mathfrak{m} H_{i}(b)\right)_{i+k+1}=0$. Hence we are done.

A graded ideal $I \subset S$ generated in degree $d$ is said to have a linear resolution if the regularity $\operatorname{reg}(I)=\max \left\{k: \beta_{i i+k}^{S}(I) \neq 0\right\}$ of $I$ is equal to $d$. Also, a graded ideal $I$ is said to be componentwise linear if the ideal $I_{\langle k\rangle}$ has linear resolution for each $k$. A monomial ideal $I \subset S$ is said to be strongly stable if $u x_{q} \in I$ implies $u x_{p} \in I$ for any $1 \leq p<q \leq n$. Note that generic initial ideals are strongly stable if $\operatorname{char}(K)=0$, and strongly stable ideals are componentwise linear.

Theorem 2.1 has a nice meaning in the special case $M=S / I$ where $I$ is a graded ideal in $S$. Let $I \subset S$ be a graded ideal and $\operatorname{Gin}(I)$ its generic initial ideal with respect to the reverse lexicographic order. It follows from [10, Theorem 1.5] that a graded ideal $I \subset S$ is componentwise linear if and only if the Betti numbers of $S / I$ reaches the upper bound given in Corollary 1.4. Also, it is not hard to show that $\alpha_{i, j}(S / I)=\alpha_{i, j}(S / \operatorname{Gin}(I))$ for all $i$ and $j$ (see [10, Lemma 2.5]). Then, since $\operatorname{Gin}(I)$ is componentwise linear, we have

$$
\beta_{i i+k}^{S}(S / \operatorname{Gin}(I))=\sum_{j=1}^{n-i+1}\left(\begin{array}{c}
n-j \\
i-1
\end{array}\right) \alpha_{j, k}(S / I) \quad \text { for all } i \text { and } k .
$$


This fact and Theorem 2.1 immediately imply

Corollary 2.3. Suppose $\operatorname{char}(K)=0$. Let $I \subset S$ be a graded ideal. If for some $i>1$ and $k \geq 0, \beta_{i i+k}^{S}(S / I)=\beta_{i i+k}^{S}(S / \operatorname{Gin}(I))$, then

$$
\beta_{q q+k}^{S}(S / I)=\beta_{q q+k}^{S}(S / \operatorname{Gin}(I)) \quad \text { for all } q \geq i .
$$

Remark 2.4. The assumption $i>1$ in Theorem 2.1 (and Corollary 2.3) is necessary. In the case when $i=1$, we notice from the proof that we need to show that $\left(\mathfrak{m} H_{2}(b)\right)_{2+k}=0$ and $\left(\mathfrak{m} H_{1}(b)\right)_{2+k}=0$ for all $b \leq n-1$. As the set $A_{1, n} \backslash\{(1, b), b \leq n-1\}=\emptyset$, the second equality does not follow. Moreover in the case when $M=S / I$ where $I \subset S$ is a graded ideal, we always have $\beta_{1 d_{0}}^{S}(S / I)=\beta_{1 d_{0}}^{S}(S / \operatorname{Gin}(I))=\sum_{j=1}^{n+1} \alpha_{j, d_{0}-1}(S / I)$ where $d_{0}$ is the minimum of the degrees of generators of $I$. So if Theorem 2.1 would have been true for $i=1$, then it would follow that $\beta_{i i+d_{0}-1}^{S}(S / I)=$ $\sum_{j=1}^{n-i+1}\left(\begin{array}{c}n-j \\ i-1\end{array}\right) \alpha_{j, d_{0}-1}(S / I)$ for all $i$, which is false in general.

As we see in Remark 2.4, Corollary 2.3 is false for $i=1$. However, the following property is true for the first graded Betti numbers.

Corollary 2.5. Suppose $\operatorname{char}(K)=0$. Let $I \subset S$ be a graded ideal. Then, for a given integer $k$, the graded Betti numbers $\beta_{i i+k}^{S}(S / I)=$ $\beta_{i i+k}^{S}(S / \operatorname{Gin}(I))$ for all $i \geq 1$ if and only if $\beta_{1, k+1}^{S}(S / I)=\beta_{1, k+1}^{S}(S / \operatorname{Gin}(I))$ and $\beta_{1, k+2}^{S}(S / I)=\beta_{1, k+2}^{S}(S / \operatorname{Gin}(I))$.

Proof. First, we will show the "if" part. Since $\beta_{1, k+1}^{S}(S / I)=$ $\beta_{1, k+1}^{S}(S / \operatorname{Gin}(I))$ and $\beta_{1, k+2}^{S}(S / I)=\beta_{1, k+2}^{S}(S / \operatorname{Gin}(I))$, Corollary 1.4 says that $\mathfrak{m} H_{1}(b)_{1+k}=0$ and $\mathfrak{m} H_{1}(b)_{2+k}=0$ for all $b \leq n-1$. Thus Lemma 2.2 says that $\mathfrak{m} H_{a}(b)_{a+k}=0$ and $\mathfrak{m} H_{a}(b)_{a+k+1}=0$ for all $(a, b)$ with $a \in \mathbb{Z}$ and $b \leq n-1$. Then, by Corollary 1.4, we have $\beta_{i i+k}^{S}(S / I)=\beta_{i i+k}^{S}(S / \operatorname{Gin}(I))$ for all $i \geq 1$.

Next, we will show the "only if" part. Since $\beta_{1, k+1}^{S}(S / I)=$ $\beta_{1, k+1}^{S}(S / \operatorname{Gin}(I))$ follows from the assumption, what we must prove is $\beta_{1, k+2}^{S}(S / I)=\beta_{1, k+2}^{S}(S / \operatorname{Gin}(I))$. Since $\beta_{2, k+2}^{S}(S / I)=\beta_{2, k+2}^{S}(S / \operatorname{Gin}(I))$, Corollary 1.4 says that $\mathfrak{m} H_{a}(b)_{(a+k+1)}=0$ for all $(a, b) \in A_{2, n} \backslash\{(2, b)$ : $b \leq n-1\}=A_{1, n}$. This fact and Corollary 1.4 imply $\beta_{1, k+2}^{S}(S / I)=$ $\beta_{1, k+2}^{S}(S / \operatorname{Gin}(I))$. 
For any monomial $u \in S$, write $m(u)$ for the maximal integer $i$ such that $x_{i}$ divides $u$. We recall a result of Eliahou-Kervaire [12] which we need in the proof of our next proposition. They proved that if $I \subset S$ is a strongly stable ideal then

$$
\beta_{i i+j}(I)=\sum_{u \in G(I), \operatorname{deg}(u)=j}\left(\begin{array}{c}
m(u)-1 \\
i
\end{array}\right) \text { for all } i \text { and } j
$$

where $G(I)$ is the set of minimal monomial generators of $I$. AramovaHerzog-Hibi [4, Theorem 1.1] proved that a graded ideal $I$ in $S$ with $\operatorname{char}(K)=0$ is componentwise linear if and only if $\beta_{i j}^{S}(I)=\beta_{i j}^{S}(\operatorname{Gin}(I))$ for all $i, j$. We will refine this result in terms of the maximal degree of minimal generators.

Proposition 2.6. Suppose $\operatorname{char}(K)=0$. Let $I \subset S$ be a graded ideal, and let $d$ be the maximum of the degrees of the generators of $I$. Then the following conditions are equivalent.

(i) I is componentwise linear;

(ii) $\beta_{i i+k}^{S}(I)=\beta_{i i+k}^{S}(\operatorname{Gin}(I))$ for all $i \geq 0$ and all $k \leq d$;

(iii) $\beta_{11+k}^{S}(I)=\beta_{11+k}^{S}(\operatorname{Gin}(I))$ for all $k \leq d$;

(iv) $\beta_{0 k}^{S}(I)=\beta_{0 k}^{S}(\operatorname{Gin}(I))$ for all $k \leq d+1$.

Proof. (i) $\Rightarrow$ (ii) follows from [4, Theorem 1.1] and (ii) $\Rightarrow$ (iii) is obvious. On the other hand, we already proved that if $\beta_{1 k}^{S}(I)=\beta_{1 k}^{S}(\operatorname{Gin}(I))$, then we have $\beta_{0 k}^{S}(I)=\beta_{0 k}^{S}(\operatorname{Gin}(I))$ in the proof of Corollary 2.5. This fact implies (iii) $\Rightarrow$ (iv).

Now we show (iv) $\Rightarrow(\mathrm{i})$. We have $\beta_{0 d+1}^{S}(I)=\beta_{0 d+1}^{S}(\operatorname{Gin}(I))=0$, by assumption. Now, since $\operatorname{Gin}(I)$ is strongly stable, by Eliahou-Kervaire formula (11) we have $\beta_{i, i+d+1}^{S}(I)=\beta_{i, i+d+1}^{S}(\operatorname{Gin}(I))=0$ for all $i \geq 0$. However, the equality of graded betti numbers $\beta_{1 d+2}^{S}(I)=\beta_{1 d+2}^{S}(\operatorname{Gin}(I))=$ 0 implies the equality $\beta_{0 d+2}^{S}(I)=\beta_{0 d+2}^{S}(\operatorname{Gin}(I))=0$ as we see in the proof of Corollary 2.5. Then again we have $\beta_{i, i+d+2}^{S}(I)=\beta_{i, i+d+2}^{S}(\operatorname{Gin}(I))=0$ for all $i \geq 0$. Arguing inductively, we have $\beta_{0 j}^{S}(I)=\beta_{0 j}^{S}(\operatorname{Gin}(I))$ for all $j \geq 0$. Then Corollary 2.5 implies that $\beta_{i j}(I)=\beta_{i j}(\operatorname{Gin}(I))$ for all $i, j$. Hence $I$ is componentwise linear. 


\section{§3. The Cartan-complex and generic annihilator numbers}

In this section, we recall some basic facts about Cartan complex introduced by Cartan and consider generic annihilator numbers in an exterior algebra.

Let $K$ be an infinite field, $V$ an $n$-dimensional $K$-vector space with basis $e_{1}, \ldots, e_{n}$ and $E=\bigoplus_{k=0}^{n} \Lambda^{k} V$ the exterior algebra of $V$. For any subset $S=\left\{i_{1}, \ldots, i_{d}\right\}$ with $1 \leq i_{1}<\cdots<i_{d} \leq n$, the element $e_{S}=e_{i_{1}} \wedge \cdots \wedge e_{i_{d}} \in$ $E$ is called a monomial of $E$ of degree $d$. Let $v_{1}, \ldots, v_{m} \in E_{1}$ be linear forms. The Cartan complex $C_{\bullet}\left(v_{1}, \ldots, v_{m} ; E\right)$ of the sequence $v_{1}, \ldots, v_{m}$ is defined as the complex whose $i$-chains $C_{i}\left(v_{1}, \ldots, v_{m} ; E\right)$ are the elements of degree $i$ of the free divided power algebra $E\left\langle x_{1}, \ldots, x_{m}\right\rangle$. Thus $C_{\bullet}\left(v_{1}, \ldots, v_{m} ; E\right)$ is the polynomial ring over $E$ in the set of variables

$$
x_{i}^{(j)}, \quad i=1,2, \ldots, m, j=1,2, \ldots,
$$

modulo the relations

$$
x_{i}^{(j)} x_{i}^{(k)}=\frac{(j+k) !}{j ! k !} x_{i}^{(k+j)},
$$

where we set $x_{i}^{(0)}=1$ and $x_{i}^{(1)}=x_{i}$ for $i=1, \ldots, m$. The algebra $C \cdot\left(v_{1}, \ldots, v_{m} ; E\right)$ is a free $E$-module with basis $x^{(a)}=x_{1}^{\left(a_{1}\right)} \cdots x_{m}^{\left(a_{m}\right)}$ with $a \in \mathbb{Z}^{m}$.

The $E$-linear differential $\partial$ on $C \cdot\left(v_{1}, \ldots, v_{m} ; E\right)$ is defined by

$$
\partial\left(x^{(a)}\right)=\sum_{a_{i}>0} v_{i} \cdot x_{1}^{\left(a_{1}\right)} \cdots x_{i}^{\left(a_{i}-1\right)} \cdots x_{m}^{\left(a_{m}\right)} .
$$

It is easily verified that $\partial \circ \partial=0$, so that $C \bullet\left(v_{1}, \ldots, v_{m} ; E\right)$ is indeed a complex.

Let $\mathcal{M}$ be the category of finitely generated graded left and right $E$ module $M$ satisfying $a x=(-1)^{\operatorname{deg}(a)+\operatorname{deg}(x)} x a$ for all homogeneous elements $a \in E$ and $x \in M$, where $M \in \mathcal{M}$. The complex $C \cdot\left(v_{1}, \ldots, v_{m} ; M\right)=$ $C_{\bullet}\left(v_{1}, \ldots, v_{m} ; E\right) \otimes_{E} M$ is called the Cartan complex of $M$ with respect to $v_{1}, \ldots, v_{m} \in E_{1}$, and its homology $H_{\bullet}\left(v_{1}, \ldots, v_{m} ; M\right)$ is called the Cartan homology. We recall two basic properties of the Cartan homology. (See [2] or [14] for the detail.)

Lemma 3.1. ([2, Theorem 2.2]) Let $v_{1}, \ldots, v_{n} \in E$ be linearly independent linear forms and $M \in \mathcal{M}$. One has

$$
H_{i}\left(v_{1}, \ldots, v_{n} ; M\right)_{j} \cong \operatorname{Tor}_{i}^{E}(K, M)_{j}
$$


Lemma 3.2. ([2, Corollary 2.4]) Let $v_{1}, \ldots, v_{n} \in E$ be linear forms and $M \in \mathcal{M}$. For $p=1,2, \ldots, n-1$, there exists a long exact sequence

$$
\begin{aligned}
\cdots & \stackrel{\gamma_{i, p}}{\longrightarrow} H_{i}\left(v_{1}, \ldots, v_{p} ; M\right) \stackrel{\eta_{i, p}}{\longrightarrow} H_{i}\left(v_{1}, \ldots, v_{p+1} ; M\right) \\
& \stackrel{\psi_{i, p}}{\longrightarrow} H_{i-1}\left(v_{1}, \ldots, v_{p+1} ; M\right)(-1) \stackrel{\gamma_{i-1, p}}{\longrightarrow} H_{i-1}\left(v_{1}, \ldots, v_{p} ; M\right) \\
& \stackrel{\eta_{i-1, p}}{\longrightarrow} H_{i-1}\left(v_{1}, \ldots, v_{p+1} ; M\right) \longrightarrow
\end{aligned}
$$

where $\eta_{i, p}$ is the map induced by the inclusion map and the maps $\psi_{i, p}$ and $\gamma_{i, p}$ are defined as follows: If $z=g_{0}+g_{1} x_{p+1}+\cdots+g_{i} x_{p+1}^{(i)}$ is a cycle in $C_{i}\left(v_{1}, \ldots, v_{p+1} ; M\right)$ with each $g_{k} \in C_{i}\left(v_{1}, \ldots, v_{p} ; M\right)$, then $\psi_{i, p}([z])=$ $\left[g_{1}+g_{2} x_{p+1}+\cdots+g_{i} x_{p+1}^{(i-1)}\right]$ and $\gamma_{i, p}([z])=\left[g_{0} v_{p+1}\right]$.

Next, we will introduce generic annihilator numbers in the exterior algebra. Let $M \in \mathcal{M}$ and let $v_{1}, \ldots, v_{n} \in E$ be generic linear forms of $M$. For $p=1,2, \ldots, n$, set

$$
A^{(p)}(M)=\left(\left(v_{1}, \ldots, v_{p-1}\right) M:_{M} v_{p}\right) /\left(v_{1}, \ldots, v_{p}\right) M
$$

and

$$
\alpha_{p, k}(M)=\operatorname{dim}_{K}\left(A^{(p)}(M)_{k}\right) .
$$

Note that $A^{(p)}(M)=\operatorname{Ker}\left(\gamma_{0, p-1}\right)$ for $p=2,3, \ldots, n$. These numbers $\alpha_{p, k}(M)$ are constant for a generic choice of linear forms $v_{1}, \ldots, v_{n} \in E_{1}$, and will be called exterior generic annihilator numbers of $M$. In the rest of this section, we will give the formula to compute the graded Betti numbers of generic initial ideals in the exterior algebra from exterior generic annihilator numbers.

A monomial ideal $J \subset E$ is said to be strongly stable if $e_{S} \in J$ and $j \in S$ implies that $e_{(S \backslash\{j\}) \cup\{i\}} \in J$ for all $i<j$ with $i \notin S$. It is known that generic initial ideals are strongly stable ([2, Proposition 1.7]).

Lemma 3.3. Let $J \subset E$ be a graded ideal. Then one has

$$
\begin{aligned}
& \alpha_{p, k}(E / J)=\left|\left\{e_{S} \in G(\operatorname{Gin}(J))_{k+1}: \max (S)=n-p+1\right\}\right| \\
& \quad \text { for } p=1,2, \ldots, n,
\end{aligned}
$$

where $|A|$ denotes the cardinality of a finite set $A$ and $G(\operatorname{Gin}(J))_{k+1}$ is the set of minimal monomial generators of $\operatorname{Gin}(J)$ of degree $k+1$. 
Proof. By a generic change of coordinates, we may assume that in $(J)=$ $\operatorname{Gin}(J)$ and $v_{1}, v_{2}, \ldots, v_{p+1}=e_{n}, e_{n-1}, \ldots, e_{n-p}$. Then, by (12), we have

$$
A^{(p+1)}(E / J)=\left(\left(e_{n}, \ldots, e_{n-p+1}\right)+J:_{E} e_{n-p}\right) /\left(\left(e_{n}, \ldots, e_{n-p}\right)+J\right),
$$

where $p=0,1, \ldots, n-1$. Set

$B^{(p+1)}(E / J)=\left(\left(e_{n}, \ldots, e_{n-p+1}\right)+\operatorname{in}(J):_{E} e_{n-p}\right) /\left(\left(e_{n}, \ldots, e_{n-p}\right)+\operatorname{in}(J)\right)$.

Since we consider the reverse lexicographic order induced by $e_{1}>\cdots>e_{n}$, it follows from [1, Proposition 5.1] that

$$
\operatorname{in}\left(\left(e_{n}, \ldots, e_{n-p+1}\right)+J:_{E} e_{n-p}\right)=\left(\left(e_{n}, \ldots, e_{n-p+1}\right)+\operatorname{in}(J):_{E} e_{n-p}\right)
$$

and

$$
\operatorname{in}\left(\left(e_{n}, \ldots, e_{n-p}\right)+J\right)=\left(e_{n}, \ldots, e_{n-p}\right)+\operatorname{in}(J) .
$$

Since $\left(\left(e_{n}, \ldots, e_{n-p+1}\right)+J:_{E} e_{n-p}\right) \supset\left(e_{n}, \ldots, e_{n-p}\right)+J$ and taking initial ideals does not change Hilbert functions, it follows that $B^{(p+1)}(E / J)$ and $A^{(p+1)}(E / J)$ have the same Hilbert function. Thus we have $\alpha_{p, k}(E / J)=$ $\operatorname{dim}_{K} B^{(p)}(E / J)_{k}$ for all $k \geq 0$.

Then, to prove the claim, it is enough to show that the set of monomials

$$
\begin{aligned}
& \left\{\left[e_{S}\right] \in E /\left(\left(e_{n}, \ldots, e_{n-p}\right)+\operatorname{in}(J)\right):\right. \\
& \left.\max (S)<n-p, e_{S} \wedge e_{n-p} \in G(\operatorname{in}(J))_{k+1}\right\}
\end{aligned}
$$

forms a $K$-basis of $B^{(p+1)}(E / J)_{k}$.

If $e_{S}$ satisfies the condition of (13), then we have $e_{S} \notin\left(e_{n}, \ldots, e_{n-p}\right)+$ $\operatorname{in}(J)$. Thus the set $(13)$ is indeed the set of $K$-linearly independent monomials belonging to $B^{(p+1)}(E / J)$. Hence we need to prove that any nonzero monomial $e_{S} \in B^{(p+1)}(E / J)$ of degree $k$ is contained in the set (13).

Let $\left[e_{S}\right] \in B^{(p+1)}(E / J) \backslash\{0\}$ be a monomial of degree $k$. Then we have $e_{S} \wedge e_{n-p} \in\left(e_{n}, \ldots, e_{n-p+1}\right)+\operatorname{in}(J)$. Also, since $\left[e_{S}\right]$ is not zero, we have $e_{S} \notin\left(e_{n}, \ldots, e_{n-p}\right)$. Thus we have $\max (S)<n-p$ and $e_{S} \wedge e_{n-p} \in \operatorname{in}(J)$. Since in $(J)=\operatorname{Gin}(J)$ is strongly stable and $e_{S} \notin \operatorname{in}(J)$, any monomial $e_{T} \in E$ of degree $k$ which divides $e_{S} \wedge e_{n-p}$ does not belongs to $\operatorname{in}(J)$. Thus we have $e_{S} \wedge e_{n-p} \in G(\operatorname{in}(J))$, and $\left[e_{S}\right]$ is contained in the set (13). 
For a monomial $e_{S} \in E$, let $m\left(e_{S}\right)=\max (S)$. If $J \subset E$ is a strongly stable ideal, then it follows from [2, Corollary 3.3] that

$$
\beta_{i i+k}^{E}(E / J)=\sum_{p=k+1}^{n} \sum_{\begin{array}{c}
e_{S} \in G(J)_{k+1} \\
m\left(e_{S}\right)=p
\end{array}}\left(\begin{array}{c}
p-1+i-1 \\
i-1
\end{array}\right)
$$

for all $i \geq 1$ and all $k \geq 0$.

Since every generic initial ideal is strongly stable, the above equality together with Lemma 3.3 imply the next lemma.

Lemma 3.4. Let $J$ be a graded ideal in E. Then one has

$$
\beta_{i i+k}^{E}(E / \operatorname{Gin}(J))=\sum_{p=1}^{n-k}\left(\begin{array}{c}
n-p+i-1 \\
i-1
\end{array}\right) \alpha_{p, k}(E / J)
$$

for all $i \geq 1$ and all $k \geq 0$.

\section{§4. Rigidity of resolutions over an exterior algebra}

In this section, we will prove similar results studied in Section 2 for generic initial ideals in an exterior algebra.

Let $M \in \mathcal{M}$. Throughout this section, let $v_{1}, \ldots, v_{n} \in E_{1}$ be generic liner forms and write $H_{i}(p)_{k}, h_{i, k}(p)$ and $\alpha_{p, k}$ for $H_{i}\left(v_{1}, \ldots, v_{p} ; M\right)_{k}, \operatorname{dim}_{K}$ $\left(H_{i}\left(v_{1}, \ldots, v_{p} ; M\right)_{k}\right)$ and $\alpha_{p, k}(M)$ respectively. Set $\delta_{i, p, k}=\operatorname{dim}_{K}\left(\operatorname{Im}\left(\gamma_{i, p}\right)_{k}\right)$ for $i>0$ and $\delta_{0, p, k}=0$ for all $p, k$.

For an integer $j \geq 0$, Lemma 3.2 yields the following exact sequence

$$
\cdots \stackrel{\gamma_{i, p}}{\longrightarrow} H_{i}(p)_{j} \stackrel{\eta_{i, p}}{\longrightarrow} H_{i}(p+1)_{j} \stackrel{\psi_{i, p}}{\longrightarrow} H_{i-1}(p+1)_{j-1} \stackrel{\gamma_{i-1, p}}{\longrightarrow} H_{i-1}(p)_{j} \longrightarrow \cdots
$$

where $p=1,2, \ldots, n-1$. Then, in the same way as Section 1 , we have

$$
h_{1, k}(p+1)=h_{1, k}(p)+\alpha_{p+1, k-1}-\delta_{1, p, k}
$$

and, for $i>1$, we have

$$
h_{i, i+k}(p+1)=h_{i, i+k}(p)+h_{i-1, i+k-1}(p+1)-\left\{\delta_{i, p, i+k}+\delta_{i-1, p, i+k}\right\} .
$$


Proposition 4.1. With the same notation as above, one has

$$
\begin{aligned}
h_{i, i+k}(p) & =\sum_{j=1}^{p}\left(\begin{array}{c}
p-j+i-1 \\
i-1
\end{array}\right) \alpha_{j, k} \\
& -\sum_{s=1}^{i} \sum_{j=1}^{p-1}\left(\begin{array}{c}
p-1-j+i-1-(s-1) \\
i-1-(s-1)
\end{array}\right)\left\{\delta_{s, j, s+k}+\delta_{s-1, j, s+k}\right\} .
\end{aligned}
$$

Proof. The proof is quite similar to the proof of Proposition 1.1. So we will skip some detail calculations.

We use induction on $p$ and $i$. First, we will show the case $p=1$. Recall that $C_{\bullet}\left(v_{1} ; M\right)$ is the complex

$$
\cdots \longrightarrow C_{i+1}\left(v_{1} ; M\right) \stackrel{\partial}{\longrightarrow} C_{i}\left(v_{1} ; M\right) \stackrel{\partial}{\longrightarrow} C_{i-1}\left(v_{1} ; M\right) \longrightarrow \cdots
$$

with the differential $\partial\left(x_{1}^{(i)}\right)=v_{1} x_{1}^{(i-1)}$. Thus we have

$$
H_{i}(1)_{i+k} \cong\left(\left(M:_{M} v_{1}\right) / v_{1} M\right)_{k}=A^{(1)}(M)_{k},
$$

and therefore we have $h_{i, i+k}(1)=\alpha_{1, k}$ for all $i \geq 1$ and all $k \geq 0$. This is equal to the formula (16).

Second, we will consider the case $i=1$. Since we already proved $h_{1,1+k}(1)=\alpha_{1, k}$, the equation (14) says that

$$
h_{1,1+k}(p)=\left\{\alpha_{1, k}+\cdots+\alpha_{p, k}\right\}-\left\{\delta_{1,1,1+k}+\cdots+\delta_{1, p-1,1+k}\right\}
$$

which is equal to the formula (16).

Finally, the formula (16) for $i>1$ and $p>1$ follows from the equation (15) together with the induction hypothesis in the same way as Proposition 1.1.

Next, we will show the following vanishing property of $\operatorname{Im}\left(\gamma_{i, p}\right)$, which is an analogue of Lemma 2.2.

LEMMA 4.2. Let $i \geq 1$ be a positive integer. If $\delta_{i, p, k}=0$ for all $1 \leq$ $p \leq n-1$, then one has $\delta_{i+t, p, k+t}=0$ for all $1 \leq p \leq n-1$ and all $t \geq 0$.

Proof. It is enough to prove the claim for $t=1$. Remark that $\delta_{i, p, k}=0$ if and only if the map $\eta_{i, p}: H_{i}(p)_{k} \rightarrow H_{i}(p+1)_{k}$ is injective. Let $\partial_{\ell}^{(p)}$ : $H_{i+1}(p)_{k+1} \rightarrow H_{i}(p)_{k}$ be the map defined by

$$
\partial_{\ell}^{(p)}\left(\left[g_{0}+g_{1} x_{\ell}+g_{2} x_{\ell}^{(2)}+\cdots+g_{i+1} x_{\ell}^{(i+1)}\right]\right)=\left[g_{1}+g_{2} x_{\ell}+\cdots+g_{i+1} x_{\ell}^{(i)}\right],
$$


where $1 \leq \ell \leq p$ and each $g_{t}$ does not contain the variable $x_{\ell}^{(s)}$ for all $s \geq 1$. Thus $\partial_{p}^{(p)}$ is equal to the map $\psi_{i+1, p-1}$ which appears in Lemma 3.2. Set $\partial^{(p)}=\bigoplus_{\ell=1}^{p} \partial_{\ell}^{(p)}$. Then we have the following commutative diagram.

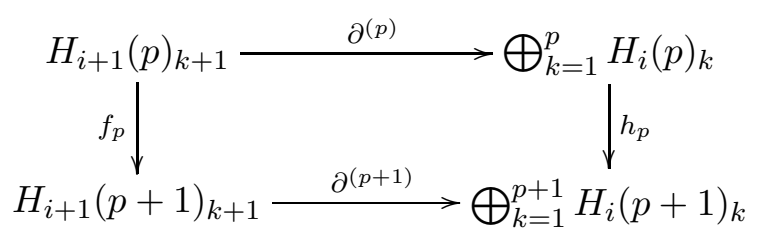

where $h_{p}$ is the map defined by $h_{p}\left(z_{1}, \ldots, z_{p}\right)=\left(\eta_{i, p}\left(z_{1}\right), \ldots, \eta_{i, p}\left(z_{p}\right), 0\right)$ and $f_{p}$ is the map defined by $f_{p}(z)=\eta_{i+1, p}(z)$.

Then $\partial^{(1)}$ is injective since $\partial^{(1)}\left(\left[g_{i+1} x_{1}^{(i+1)}\right]\right)=\left[g_{i+1} x_{1}^{(i)}\right]$. Also, by the assumption, the map $\eta_{i, p}: H_{i}(p)_{k} \rightarrow H_{i+1}(p+1)_{k}$ is injective for all $1 \leq p \leq n-1$. Thus $h_{p}$ is injective for all $1 \leq p \leq n-1$. We will show that if $\partial^{(p)}$ is injective then $\partial^{(p+1)}$ is also injective.

Set $u \in \operatorname{Ker}\left(\partial^{(p+1)}\right)$. Then we have $\partial_{p+1}^{(p+1)}(u)=\psi_{i+1, p}(u)=0$. Thus, by the long exact sequence in Lemma 3.2, there exists $w \in H_{i+1}(p)$ such that we have $\eta_{i+1, p}(w)=f_{p}(w)=u$. Since $h_{p} \circ \partial^{(p)}(w)=\partial^{(p+1)} \circ f_{p}(w)=0$ and $h_{p} \circ \partial^{(p)}$ is injective by the induction hypothesis, it follows that $w=0$ and $\partial^{(p+1)}$ is injective.

Now, we proved that $\partial^{(p)}$ is injective for all $1 \leq p \leq n-1$. Thus $h_{p} \circ \partial^{(p)}$ is injective for all $1 \leq p \leq n-1$. This fact together with the commutative diagram imply that the map $\eta_{i+1, p}: H_{i+1}(p)_{k+1} \rightarrow H_{i+1}(p+1)_{k+1}$ is injective for all $1 \leq p \leq n-1$. Hence we have $\delta_{i+1, p, k+1}=\operatorname{dim}_{K}\left(\operatorname{Im}\left(\gamma_{i+1, p}\right)_{k+1}\right)=0$ for all $1 \leq p \leq n-1$.

Proposition 4.1 and Lemma 4.2 imply the next theorem.

TheOrem 4.3. Let $M \in \mathcal{M}$. Suppose that for some $i>1$ and $k \geq 0$, we have $\beta_{i i+k}^{E}(M)=\sum_{j=1}^{n}\left(\begin{array}{c}n-j+i-1 \\ i-1\end{array}\right) \alpha_{j, k}(M)$. Then

$$
\beta_{q q+k}^{E}(M)=\sum_{j=1}^{n}\left(\begin{array}{c}
n-j+i-1 \\
i-1
\end{array}\right) \alpha_{j, k}(M) \quad \text { for all } q \geq 1 .
$$

Proof. Since all binomial coefficients in the formula (16) are nonzero, the assumption says that $\delta_{s, p, s+k}=0$ and $\delta_{s-1, p, s+k}=0$ for all $1 \leq s \leq i$ and all $1 \leq p \leq n-1$. Then Lemma 4.2 says that $\delta_{s, p, s+k}=0$ and $\delta_{s-1, p, s+k}=0$ for all $s \geq 1$ and all $1 \leq p \leq n-1$. Thus, the statement follow from the formula (16). 
Next we consider the case $M=E / J$. Lemma 3.3 says that, for any graded ideal $J$ of $E$, one has $\alpha_{j, k}(E / J)=0$ for $j>n-k$. Thus for any $i \geq 1$ and $k \geq 0$ we have $\sum_{j=1}^{n}\left(\begin{array}{c}n-j+i-1 \\ i-1\end{array}\right) \alpha_{j, k}(E / J)=\sum_{j=1}^{n-k}\left(\begin{array}{c}n-j+i-1 \\ i-1\end{array}\right) \alpha_{j, k}(E / J)$. Then the following corollaries follows from Lemma 3.4 and Theorem 4.3 in the same way as in Section 2.

Corollary 4.4. Let $J \subset E$ be a graded ideal. If $\beta_{i i+k}^{E}(E / J)=$ $\beta_{i i+k}^{E}(E / \operatorname{Gin}(J))$ for some $i>1$ and $k \geq 0$, then

$$
\beta_{q q+k}^{E}(E / J)=\beta_{q q+k}^{E}(E / \operatorname{Gin}(J)) \quad \text { for all } q \geq 1 .
$$

Corollary 4.5. Let $J \subset E$ be a graded ideal. Then, for a given integer $k$, the graded Betti numbers $\beta_{i i+k}^{E}(E / J)=\beta_{i i+k}^{E}(E / \operatorname{Gin}(J))$ for all $i \geq 1$ if and only if $\beta_{1, k+1}^{E}(E / J)=\beta_{1, k+1}^{E}(E / \operatorname{Gin}(J))$ and $\beta_{1, k+2}^{E}(E / J)=$ $\beta_{1, k+2}^{E}(E / \operatorname{Gin}(J))$.

Remark 4.6. Notice that the above Corollary 4.4 and Corollary 2.3 in Section 2 are similar. But as we see Corollary 4.4 is relatively more stronger. We give here an example to show that in the case of a polynomial ring one cannot have the stronger result as in Corollary 4.4. Consider the ideal $I=\left(x_{1} x_{4}^{2}, x_{2}^{3}, x_{2}^{2} x_{3}\right) \subset S=\mathbb{C}\left[x_{1}, x_{2}, x_{3}, x_{4}\right]$. The minimal graded free resolution of $S / I$ and $S / \operatorname{Gin}(I)$ are given by:

$$
0 \longrightarrow S(-7) \longrightarrow S(-4) \oplus S^{2}(-6) \longrightarrow S^{3}(-3) \longrightarrow S \longrightarrow S / I \longrightarrow 0,
$$

and

$$
\begin{aligned}
0 \longrightarrow S(-7) \longrightarrow & S^{2}(-4) \oplus S(-5) \oplus S^{2}(-6) \longrightarrow \\
& S^{3}(-3) \oplus S(-4) \oplus S(-5) \longrightarrow S \longrightarrow S / \text { Gin } I \longrightarrow 0 .
\end{aligned}
$$

From above resolutions, we see that $\beta_{2,2+4}^{S}(S / I)=\beta_{2,2+4}^{S}(S / \operatorname{Gin}(I))=2$ and ofcourse then $\beta_{3,3+4}^{S}(S / I)=\beta_{3,3+4}^{S}(S / \operatorname{Gin}(I))=1$. But the graded Betti number $\beta_{1,1+4}^{S}(S / I)=0 \neq 1=\beta_{1,1+4}^{S}(S / \operatorname{Gin}(I))$.

In the case of exterior algebra, the notions of regularity, linear resolutions and componentwise linear ideals are defined in the same way as in the case of polynomial ring. In [4, Theorem 2.1] it was proved that a graded ideal $J$ in $E$ is componentwise linear if and only if $J$ and $\operatorname{Gin}(J)$ have the same graded Betti numbers. Theorem 4.4 and Corollary 4.5 provide the following new characterization of componentwise linear ideals in the exterior algebra. (See also [19] for other characterizations of componentwise linear ideals.) 
TheOREM 4.7. A graded ideal $J$ in the exterior algebra $E$ is componentwise linear if and only if $\beta_{i}^{E}(E / J)=\beta_{i}^{E}(E / \operatorname{Gin}(J))$ for some $i \geq 1$.

Proof. Since $\beta_{i i+k}^{E}(E / J) \leq \beta_{i i+k}^{E}(E / \operatorname{Gin}(J))$ for all $i \geq 1$ and $k \geq 0$, the equality $\beta_{i}^{E}(E / J)=\beta_{i}^{E}(E / \operatorname{Gin}(J))$ implies $\beta_{i i+k}^{E}(E / J)=$ $\beta_{i i+k}^{E}(E / \operatorname{Gin}(J))$ for all $k \geq 0$. Then Theorem 4.4 and Corollary 4.5 say that $\beta_{i}^{E}(E / J)=\beta_{i}^{E}(E / \operatorname{Gin}(J))$ for some $i \geq 1$ if and only if $J$ and $\operatorname{Gin}(J)$ have the same graded Betti numbers. Hence the claim follows.

\section{$\S 5$. Linear components and graded Betti numbers}

Throughout this section, we assume that $R$ is either the polynomial ring $S$ over the field $K$ with $\operatorname{char}(K)=0$ or the exterior algebra $E$ over an infinite field.

First, we will extend Corollaries 2.3 and 4.4 to lexsegment ideals and generic initial ideals with respect to any term order. For a strongly stable ideal $I$ in $R$ and for integers $q=1, \ldots, n$ and $k \geq 0$, let

$$
m_{\leq q}(I, k)=\mid\{u \in I: u \text { is a monomial with } m(u) \leq q \text { and } \operatorname{deg}(u)=k\} \mid .
$$

Lemma 5.1. Let $I \subset R$ be a graded ideal and $I^{\prime} \subset R$ a strongly stable ideal with the same Hilbert function as $I$. Assume that $I^{\prime}$ satisfies $m_{\leq q}\left(I^{\prime}, d\right) \leq m_{\leq q}(\operatorname{Gin}(I), d)$ for all $q, d$ and $\beta_{i i+k}^{R}(R / I)=\beta_{i i+k}^{R}\left(R / I^{\prime}\right)$ for some $i>1$ and $k \geq 0$.

(i) If $R=S$, then one has $\beta_{q q+k}^{S}(S / I)=\beta_{q q+k}^{S}\left(S / I^{\prime}\right)$ for all $q \geq i$.

(ii) If $R=E$, then one has $\beta_{q q+k}^{E}(E / I)=\beta_{q q+k}^{E}\left(E / I^{\prime}\right)$ for all $q \geq 1$.

Proof. We will show the case $R=S$. (The proof for the case $R=E$ is same.) It follows from [7, Proposition 2.3] that, for any strongly stable ideal $J \subset S$, we have

$$
\begin{aligned}
\beta_{i i+j}^{S}(S / J)= & \operatorname{dim}_{K} J_{j+1}\left(\begin{array}{c}
n-1 \\
i
\end{array}\right) \\
& -\sum_{q=i}^{n-1} m_{\leq q}(J, j+1)\left(\begin{array}{c}
k-1 \\
i-1
\end{array}\right)-\sum_{q=i+1}^{n} m_{\leq q}(J, j)\left(\begin{array}{c}
k-1 \\
i
\end{array}\right)
\end{aligned}
$$

for all $i$ and $j$. (A similar formula for graded Betti numbers over the exterior algebra appears in [2, Theorem 4.4].) Then by (17) and the assumption, 
we have $\beta_{i j}^{S}(S / I) \leq \beta_{i j}^{S}(S / \operatorname{Gin}(I)) \leq \beta_{i j}^{S}\left(S / I^{\prime}\right)$ for all $i, j$. Thus, by Corollary 2.3, what we must prove is $\beta_{q q+k}^{S}(S / \operatorname{Gin}(I))=\beta_{q q+k}^{S}\left(S / I^{\prime}\right)$ for all $q \geq i$. However (17) and the assumption imply that $m_{\leq q}(\operatorname{Gin}(I), k+1)=$ $m_{\leq q}\left(I^{\prime}, k+1\right)$ for all $q \geq i$ and $m_{\leq q}(\operatorname{Gin}(I), k)=m_{\leq q}\left(I^{\prime}, k\right)$ for all $q \geq i+1$. Hence for all $q \geq i$, we have $\beta_{q q+k}^{S}(S / \operatorname{Gin}(I))=\beta_{q q+k}^{S}\left(S / I^{\prime}\right)$ as desired.

Let $I \subset R$ be a graded ideal. We write $\operatorname{Lex}(I) \subset R$ for the unique lexsegment ideal of $R$ with the same Hilbert function as $I$ defined in [7] (or [3] for the exterior case) and $\operatorname{Gin}_{\sigma}(I)$ for the generic initial ideal of $I$ with respect to a term order $\sigma$. It is known that $\operatorname{Lex}(I)$ and $\operatorname{Gin}_{\sigma}(I)$ satisfy the assumption of Lemma 5.1 (see $[9, \S 5]$ and $[19, \S 5]$ ). Thus we have

TheOREM 5.2. Let $I \subset R$ be a graded ideal, $\sigma$ a term order and let $J$ be either $\operatorname{Gin}_{\sigma}(I)$ or $\operatorname{Lex}(I)$. Suppose that $\beta_{i i+k}^{R}(R / I)=\beta_{i i+k}^{R}(R / J)$ for some $i>1$.

(i) If $R=S$, then one has $\beta_{q q+k}^{S}(S / I)=\beta_{q q+k}^{S}(S / J)$ for all $q \geq i$.

(ii) If $R=E$, then one has $\beta_{q q+k}^{E}(E / I)=\beta_{q q+k}^{E}(E / J)$ for all $q \geq 1$.

Next, we consider when a graded ideal $J$ satisfies $\beta_{i i+d}^{E}(E / J)=$ $\beta_{i i+d}^{E}(E / \operatorname{Gin}(J))$ for all $i \geq 1$, where we fix an integer $d \geq 0$. The next lemma follows from [5] and [1, Theorem 5.3].

Lemma 5.3. Let $I \subset R$ be a graded ideal. Then, I has a linear resolution if and only if $\operatorname{Gin}(I)$ has a linear resolution.

We also require the following.

Lemma 5.4. (Crystallization Principle) Let $I \subset R$ be a graded ideal. If $I$ is generated by elements of degree $\leq d$ and $\beta_{1 d+1}^{R}(R / \operatorname{Gin}(I))=0$, then $\operatorname{reg}(I) \leq d$

The Crystallization Principle was proved by Green [13, Corollary 2.28] for generic initial ideals over a polynomial ring, however, this fact can also be proved for generic initial ideals over an exterior algebra in the same way.

Proposition 5.5. Let $I \subset R$ be a graded ideal. The following conditions are equivalent.

(i) $I_{\langle k\rangle}$ has a linear resolution; 
(ii) $\beta_{1 k+1}^{R}(R / I)=\beta_{1 k+1}^{R}(R / \operatorname{Gin}(I))$, that is, the number of elements of degree $k+1$ belonging to the set of minimal generators of $I$ is equal to that of $\operatorname{Gin}(I)$.

Proof. Let $\mathfrak{m}$ be the maximal ideal of $R$. Since $\beta_{1 k+1}^{R}(R / I)$ is the numbers of generators in $G(I)$ of degree $k+1$, we have

$$
\begin{aligned}
\beta_{1 k+1}^{R}(R / I) & =\operatorname{dim}_{K} I_{k+1}-\operatorname{dim}_{K}\left(\mathfrak{m} I_{\langle k\rangle}\right)_{k+1} \\
& =\operatorname{dim}_{K} I_{k+1}-\operatorname{dim}_{K}\left(I_{\langle k\rangle}\right)_{k+1}
\end{aligned}
$$

and

$$
\beta_{1 k+1}^{R}(R / \operatorname{Gin}(I))=\operatorname{dim}_{K}\left(\operatorname{Gin}(I)_{k+1}\right)-\operatorname{dim}_{K}\left(\mathfrak{m} \operatorname{Gin}\left(I_{\langle k\rangle}\right)\right)_{k+1} .
$$

Then, from above equations we have $\beta_{1 k+1}^{R}(R / I)=\beta_{1 k+1}^{R}(R / \operatorname{Gin}(I))$ if and only if $\operatorname{dim}_{K}\left(I_{\langle k\rangle}\right)_{k+1}=\operatorname{dim}_{K}\left(\mathfrak{m} \operatorname{Gin}\left(I_{\langle k\rangle}\right)\right)_{k+1}$.

Suppose $I_{\langle k\rangle}$ has a linear resolution. Then, by Lemma $5.3 \operatorname{Gin}\left(I_{\langle k\rangle}\right)$ has a linear resolution. Hence

$$
\operatorname{dim}_{K}\left(\mathfrak{m} \operatorname{Gin}\left(I_{\langle k\rangle}\right)\right)_{k+1}=\operatorname{dim}_{K}\left(\operatorname{Gin}\left(I_{\langle k\rangle}\right)\right)_{k+1}=\operatorname{dim}_{K}\left(I_{\langle k\rangle}\right)_{k+1} .
$$

Hence we have $\beta_{1 k+1}^{R}(R / I)=\beta_{1 k+1}^{R}(R / \operatorname{Gin}(I))$ as required. On the other hand, if $\beta_{1 k+1}^{R}(R / I)=\beta_{1 k+1}^{R}(R / \operatorname{Gin}(I))$, then $\operatorname{dim}_{K}\left(\mathfrak{m} \operatorname{Gin}\left(I_{\langle k\rangle}\right)\right)_{k+1}=$ $\operatorname{dim}_{K}\left(\operatorname{Gin}\left(I_{\langle k\rangle}\right)\right)_{k+1}$. This implies $\beta_{1 k+1}^{R}\left(R / \operatorname{Gin}\left(I_{\langle k\rangle}\right)\right)=0$. Then the Crystallization Principle says that $I_{\langle k\rangle}$ has a linear resolution.

Now, Theorem 0.1 immediately follows from the above proposition together with Corollaries 2.5 and 4.5. Indeed, (i) $\Leftrightarrow$ (iii) of Theorem 0.1 follows from Corollaries 2.5 and 4.5. Also, (ii) $\Leftrightarrow$ (iii) of Theorem 0.1 follows from Proposition 5.5.

ExAmple 5.6. Let $I=\left(x_{1}^{2}, x_{2}^{2}, x_{1} x_{2} x_{3}^{2}, x_{3}^{5}\right) \subset S=\mathbb{C}\left[x_{1}, x_{2}, x_{3}\right]$. Then we have

$$
\operatorname{Gin}(I)=\left(x_{1}^{2}, x_{1} x_{2}, x_{2}^{3}, x_{2}^{2} x_{3}^{2}, x_{1} x_{3}^{4}, x_{2} x_{3}^{5}, x_{3}^{6}\right) .
$$

Then Proposition 5.5 says that $I_{\langle k\rangle}$ has a linear resolution for $k=3,4,7,8$, $9, \ldots$ In particular, for $k=4,8,9,10, \ldots$, we have $\beta_{i i+k}^{S}(I)=\beta_{i i+k}^{S}(\operatorname{Gin}(I))$ for all $i \geq 0$. 


\section{§6. The Cancellation Principle}

Let $K$ be a field of characteristic 0 . In this section, we will study the relation between our results in Section 1 and the Cancellation Principle for generic initial ideals, which was considered in [13]. This observation would help us to understand why we require the assumption $i>1$ in Corollary 2.3 and why we need to consider $I_{\langle k\rangle}$ and $I_{\langle k+1\rangle}$ in Theorem 0.1 .

First, we recall what is the Cancellation Principle.

Lemma 6.1. ([13, Corollary 1.21]) Let I be a graded ideal in $S$ and $\sigma$ a term order. The minimal free resolution of $I$ is obtained from that of $\operatorname{in}_{\sigma}(I)$ by cancelling adjacent terms, in other words, there exists integers $\tau_{i, i+k}$ with $1 \leq i \leq n-1$ and $k \geq 0$ such that

$$
\beta_{i i+k}^{S}\left(\operatorname{in}_{\sigma}(I)\right)=\beta_{i i+k}^{S}(I)+\tau_{i, i+k}+\tau_{i+1, i+k} \quad \text { for all } i \geq 0 \text { and all } k \geq 0
$$

where we let $\tau_{0, k}=0$ for all $k \geq 0$.

We refer the reader to [13, Example 1.35] for further information about the Cancellation Principle.

Let $I$ be a graded ideal in $S$. Then Lemma 6.1 says that there exists integers $c_{i, i+k}(I)$ with $1 \leq i \leq n-1$ and with $k \geq 0$ such that

$$
\beta_{i i+k}^{S}(\operatorname{Gin}(I))=\beta_{i i+k}^{S}(I)+c_{i, i+k}(I)+c_{i+1, i+k}(I)
$$

for all $i \geq 0$ and all $k \geq 0$,

where we let $c_{0, k}(I)=0$ for all $k \geq 0$. It can be easily verified that the integers $c_{i, i+k}(I)$ are uniquely determined for a given ideal $I$. We will call the integer $c_{i, i+k}(I)$ the $(i, i+k)$-th cancellation number of $I$.

\section{EXAMPLE 6.2. Let}

$$
I=\left(x_{1}^{3}, x_{1}^{2} x_{2}, x_{1} x_{2}^{2}, x_{2}^{3}, x_{1}^{2} x_{3}, x_{1} x_{3} x_{4}\right) \subset S=\mathbb{C}\left[x_{1}, x_{2}, x_{3}, x_{4}\right] .
$$

Then we have $\operatorname{Gin}(I)=\left(x_{1}^{3}, x_{1}^{2} x_{2}, x_{1} x_{2}^{2}, x_{2}^{3}, x_{1}^{2} x_{3}, x_{1} x_{2} x_{3}, x_{1} x_{3}^{3}\right)$. The minimal free resolution of $I$ is

$$
0 \longrightarrow S(-5) \oplus S(-6) \longrightarrow S^{6}(-4) \oplus S(-5) \longrightarrow S^{6}(-3) \longrightarrow I \longrightarrow 0
$$


and that of $\operatorname{Gin}(I)$ is

$$
\begin{aligned}
0 \longrightarrow S^{2}(-5) \oplus S(-6) \longrightarrow S^{7}(-4) \oplus S^{2}(-5) \longrightarrow S^{6}(-3) \oplus S(-4) & \\
& \longrightarrow \operatorname{Gin}(I) \longrightarrow 0
\end{aligned}
$$

Hence we have $c_{1,4}(I)=1, c_{2,5}(I)=1$ and all other cancellation numbers of $I$ are 0 .

In Section 2, we already proved that (see Proposition 1.1 and (10))

$$
\begin{aligned}
\beta_{i i+k}^{S}(I)= & \beta_{i i+k}^{S}(\operatorname{Gin}(I)) \\
& -\sum_{(a, b) \in A_{i+1, n}}\left[\left(\begin{array}{c}
n-b-1 \\
i-a+1
\end{array}\right) \delta_{a, b, a+k-1}+\left(\begin{array}{c}
n-b-1 \\
i-a
\end{array}\right) \delta_{a, b, a+k}\right],
\end{aligned}
$$

where $\delta_{a, b, a+k}=\operatorname{dim}_{K}\left(\operatorname{Im} \varphi_{a, b}\right)_{a+k}$ and where $\varphi_{a, b}$ is the map which appears in the long exact sequence (1). This formula enables us to write the cancellation numbers in terms of the Koszul homology of generic linear forms.

LEMMA 6.3. With the same notation as above, one has

$$
\begin{aligned}
& c_{i, i+k}(I)= \sum_{(a, b) \in A_{i+1, n}}\left(\begin{array}{c}
n-b-1 \\
i-a
\end{array}\right) \operatorname{dim}_{K}\left(\operatorname{Im} \varphi_{a, b}\right)_{a+k} \\
& \text { for all } i \geq 0 \text { and all } k \geq 0 .
\end{aligned}
$$

Proof. For all $i \geq 0$ and all $k \geq 0$, we set $C_{i, i+k}=\sum_{(a, b) \in A_{i+1, n}}\left(\begin{array}{c}n-b-1 \\ i-a\end{array}\right)$ $\operatorname{dim}_{K}\left(\operatorname{Im} \varphi_{a, b}\right)_{a+k}$ and $C_{i, i+k}^{\prime}=\sum_{(a, b) \in A_{i+1, n}}\left(\begin{array}{c}n-b-1 \\ i-a+1\end{array}\right) \operatorname{dim}_{K}\left(\operatorname{Im} \varphi_{a, b}\right)_{a+k-1}$. Then we have

$$
\beta_{i i+k}^{S}(I)=\beta_{i i+k}^{S}(\operatorname{Gin}(I))-C_{i, i+k}-C_{i, i+k}^{\prime}
$$

Notice that we only need to show that $C_{i, i+k}^{\prime}=C_{i+1, i+k}$. Recall that, in the proof of Theorem 2.1, we already proved that

$$
A_{i+2, n} \backslash\{(i+2, b): b \leq n-1\}=A_{i+1, n} \backslash\{(i-n+b+1, b): b \leq n-1\} .
$$

Now, since the binomial $\left(\begin{array}{c}n-b-1 \\ i-a+1\end{array}\right)=0$ for all $(a, b) \in\{(i+2, b): b \leq n-1\}$ 
and for all $(a, b) \in\{(i-n+b+1, b): b \leq n-1\}$, we have

$$
\begin{aligned}
C_{i+1, i+k} & =\sum_{(a, b) \in A_{i+2, n}}\left(\begin{array}{c}
n-b-1 \\
i-a+1
\end{array}\right) \operatorname{dim}_{K}\left(\operatorname{Im} \varphi_{a, b}\right)_{a+k-1} \\
& =\sum_{(a, b) \in A_{i+2, n} \backslash\{(i+2, b): b \leq n-1\}}\left(\begin{array}{c}
n-b-1 \\
i-a+1
\end{array}\right) \operatorname{dim}_{K}\left(\operatorname{Im} \varphi_{a, b}\right)_{a+k-1} \\
& =\sum_{(a, b) \in A_{i+1, n} \backslash\{(i-n+b+1, b): b \leq n-1\}}\left(\begin{array}{c}
n-b-1 \\
i-a+1
\end{array}\right) \operatorname{dim}_{K}\left(\operatorname{Im} \varphi_{a, b}\right)_{a+k-1} \\
& =\sum_{(a, b) \in A_{i+1, n}}\left(\begin{array}{c}
n-b-1 \\
i-a+1
\end{array}\right) \operatorname{dim}_{K}\left(\operatorname{Im} \varphi_{a, b}\right)_{a+k-1} \\
& =C_{i, i+k}^{\prime} .
\end{aligned}
$$

This concludes the proof.

By using Lemma 6.3, we can prove an analogue of Corollaries 2.3 and 2.5 .

THEOREM 6.4. Let I be a graded ideal in $S$. If $c_{i, i+k}(I)=0$ for some $i \geq 1$ and $k \geq 0$, then one has $c_{q, q+k}(I)=0$ for all $q \geq i$.

Proof. It suffices to show the case $q=i+1$. Remark that $\operatorname{dim}_{K}\left(\operatorname{Im} \varphi_{a, b}\right)_{a+k}=0$ if and only if $\left(\mathfrak{m} H_{a}(b)\right)_{a+k}=0$. In the proof of Theorem 2.1, we proved that if $\operatorname{dim}_{K}\left(\operatorname{Im} \varphi_{a, b}\right)_{a+k}=0$ for all $(a, b) \in$ $A_{i+1, n} \backslash\{(i+1, b): b \leq n-1\}$, then $\operatorname{dim}_{K}\left(\operatorname{Im} \varphi_{a, b}\right)_{a+k}=0$ for all $(a, b) \in$ $A_{i+2, n} \backslash\{(i+2, b): b \leq n-1\}$. Then, since $\left(\begin{array}{c}n-b-1 \\ i-a+1\end{array}\right)=0$ for any $(a, b) \in$ $\{(i+2, b): b \leq n-1\}$, Lemma 6.3 says that $c_{i+1, i+1+k}(I)=0$.

Corollary 6.5. Let $I$ be a graded ideal in $S$. Then $c_{i, i+k}(I)=0$ for all $i \geq 1$ if and only if $I_{\langle k\rangle}$ has a linear resolution.

Proof. Since the graded Betti number $\beta_{0, k+1}^{S}(\operatorname{Gin}(I))=\beta_{0, k+1}^{S}(I)+$ $c_{1,1+k}(I)$, we have $\beta_{0, k+1}^{S}(\operatorname{Gin}(I))=\beta_{0, k+1}^{S}(I)$ if and only if $c_{1,1+k}(I)=0$. However, by Theorem 6.4, we have $c_{1,1+k}(I)=0$ if and only if $c_{i, i+k}(I)=0$ for all $i \geq 1$. Also, by Proposition 5.5, we have $\beta_{0, k+1}^{S}(\operatorname{Gin}(I))=\beta_{0, k+1}^{S}(I)$ if and only if $I_{\langle k\rangle}$ has a linear resolution. Thus the assertion follows. 
Observe that Theorems 6.4 and Corollary 6.5 are stronger than Corollaries 2.3 and 2.5. Indeed, Corollary 2.3 immediately follows from Theorem 6.4, since the graded Betti numbers $\beta_{i i+k}^{S}(I)=\beta_{i i+k}^{S}(\operatorname{Gin}(I))$ if and only if $c_{i, i+k}(I)=0$ and $c_{i+1, i+k}(I)=0$.

We also remark the next fact which follows from Lemma 6.3.

Corollary 6.6. Let I be a graded ideal in $S$. Assume that $I_{\langle k\rangle}$ has a linear resolution.

(i) If $\beta_{q, q+k+2}^{S}(I)=\beta_{q, q+k+2}^{S}(\operatorname{Gin}(I))$, then $\beta_{q+1, q+k+2}^{S}(I)=\beta_{q+1, q+k+2}^{S}(\operatorname{Gin}(I)) ;$

(ii) If $\beta_{q, q+k-1}^{S}(I)=\beta_{q, q+k-1}^{S}(\operatorname{Gin}(I))$, then $\beta_{q-1, q+k-1}^{S}(I)=\beta_{q-1, q+k-1}^{S}(\operatorname{Gin}(I))$.

Proof. By Corollary 6.5, we have $c_{\ell, \ell+k}(I)=0$ for all integers $\ell \geq 1$. Then, we have the graded Betti numbers $\beta_{q+1, q+k+2}^{S}(\operatorname{Gin}(I))=$ $\beta_{q+1, q+k+2}^{S}(I)+c_{q+1, q+k+2}(I)$ and $\beta_{q-1, q+k-1}^{S}(\operatorname{Gin}(I))=\beta_{q-1, q+k-1}^{S}(I)+$ $c_{q, q+k-1}(I)$. On the other hand, if the graded Betti number $\beta_{q, q+k+2}^{S}(I)=$ $\beta_{q, q+k+2}^{S}(\operatorname{Gin}(I))$ then we have $c_{q+1, q+k+2}(I)=0$. Also, if $\beta_{q, q+k-1}^{S}(I)=$ $\beta_{q, q+k-1}^{S}(\operatorname{Gin}(I))$ then we have $c_{q, q+k-1}(I)=0$. Thus the assertion follows.

As for any graded ideal $I, I_{\langle 1\rangle}$ always has a linear resolution, it follows that if $\beta_{q, q+3}^{S}(\operatorname{Gin}(I))=\beta_{q, q+3}^{S}(I)$ then we have $\beta_{q+1, q+3}^{S}(\operatorname{Gin}(I))=$ $\beta_{q+1, q+3}^{S}(I)$.

Since it is not difficult to find the Betti numbers of a strongly stable ideal $J$, one may expect to find all possible Betti numbers of graded ideals $I$ such that $\operatorname{Gin}(I)=J$ by using Betti numbers of $J$ and by considering all possible cancellations. However, this problem is far reaching as pointed out in [13, Example 1.35].

Thanks. All of the examples that we have presented in the paper are computed by the computer algebra system CoCoA [8]. We also mention that computations of generic initial ideals are done by a random choice of matrices. 


\section{REFERENCES}

[1] A. Aramova and J. Herzog, Almost regular sequences and Betti numbers, Amer. J. Math., 122 (2000), 689-719.

[2] A. Aramova, J. Herzog and T. Hibi, Gotzmann theorems for exterior algebras and combinatorics, J. Alg., 191 (1997), 174-211.

[3] A. Aramova, J. Herzog and T. Hibi, Squarefree lexsegment ideals, Math. Z., 228 (1998), 353-378.

[4] A. Aramova, J. Herzog and T. Hibi, Ideals with stable Betti numbers, Adv. Math., 152 (2000), no. 1, 72-77.

[5] D. Bayer and M. Stillman, A criterion for detecting $m$-regularity, Invent. Math., 87 (1987), 1-11.

[6] W. Bruns and J. Herzog, Cohen-Macaulay rings, Revised Edition, Cambridge University Press, Cambridge, 1996.

[7] A. M. Bigatti, Upper bound for the Betti numbers of a given Hilbert function, Comm. in Alg., 21 (1993), 2317-2334.

[8] CoCoA Team, CoCoA: a system for doing Computations in Commutative Algebra, available at http://cocoa.dima.unige.it.

[9] A. Conca, Koszul homology and extremal property of Gin and Lex, Trans. Amer. Math. Soc., 256 (2004), no. 7, 2945-2961.

[10] A. Conca, J. Herzog and T. Hibi, Rigid resolutions and big Betti numbers, Comment. Math. Helv., 79 (2004), 826-839.

[11] D. Eisenbud, Commutative Algebra with a View Toward Algebraic Geometry, Graduate Texts in Math., Springer-Verlag: New-York, 1995.

[12] S. Eliahou and M. Kervaire, Minimal resolutions of some monomial ideals, J. of Algebra, 129 (1990), 1-25.

[13] M. Green, Generic initial ideals, Six Lectures on Commutative Algebra (J. Elias, J. M. Giral, R. M. Miró-Roig, and S. Zarzuela, eds.), Progress in Math., 166, Birkhäuser, Basel, 1998, pp. 119-186.

[14] J. Herzog, Generic initial ideals and graded Betti numbers, Computational Commutative Algebra and Combinatorics (T. Hibi, ed.), Advanced Studies in Pure Math., Volume 33, 2002, pp. 75-120.

[15] J. Herzog and T. Hibi, Componentwise linear ideals, Nagoya Math. J., 153 (1999), $141-153$.

[16] S. Murai and T. Hibi, Gin and Lex of certain monomial ideals, Math. Scand., 99 (2006), no. 1, 76-86.

[17] V. Kodiyalam, Homological Invariants of Powers of an Ideal, Proc. Amer. Math. Soc., 118 (1993), no. 3, 757-763.

[18] V. Kodiyalam, Asymptotic Behavior of Castelnuovo-Mumford Regularity, Proc. Amer. Math. Soc., 128 (1999), no. 2, 407-411.

[19] U. Nagel, T. Römer and N. P. Vinai, Algebraic shifting and exterior and symmetric algebra methods, Comm. Algebra, 36 (2008), no. 1, 208-231. 
Satoshi Murai

Department of Pure and Applied Mathematics

Graduate School of Information Science and Technology

Osaka University

Toyonaka

Osaka, 560-0043

Japan

s-murai@ist.osaka-u.ac.jp

Pooja Singla

FB Mathematik

Universität Duisburg-Essen

Campus Essen, 45117 Essen

Germany

pooja.singla@uni-duisburg-essen.de 